\title{
CHEMICAL PROPERTIES OF HAWTHORN (Crataegus L. spp.) TAXA NATURALLY DISTRIBUTED IN WESTERN ANATOLIA PART OF TURKEY
}

\section{KEMIJSKA SVOJSTVA SVOJTI GLOGA (Crataegus L. spp.) PRIRODNO RASPROSTRANJENIH U ZAPADNOJ ANATOLIJI U TURSKOJ}

Sevgin ÖZDERIN ${ }^{* 1}$ Hüseyin FAKIR ${ }^{2}$ I.Emrah DÖNMEZ

\begin{abstract}
Summary
Chemical properties of Crataegus pentagyna subsp. pentagyna, C. orientalis subsp. orientalis, C. orientalis subsp. szovitsii, C. tanacetifolia, C. azarolus var. aronia, C. monogyna var. lasiocarpa, C. monogyna var. monogyna taxa that are naturally distributed in Western Anatolia were determined in this study. Leaf and flower samples collected from Izmit, Sakarya, Balıkesir, Izmir, Kütahya, Muğla and Isparta provinces of Western Anatolia to determine volatile components in 2010-2014 period were dried at room temperature. Volatile components that were obtained by dry phase microextraction (SPME) method in Süleyman Demirel University Central Laboratories were determined in Gas chromatography-mass spectrometry (GC-MS). A total of 81 volatile components belonging to 7 hawthorn taxa were determined. Volatile oil components that were identified at highest ratios were benzaldehyde (82.54\%) butyraldehyde (38.27\%) and (E)2-hexenal (21.67\%) components.

Moisture values of hawthorn seeds samples that were collected from sample areas during ripening period were determined. Fatty acid composition was determined in with Gas Chromatography-Flame Ionization Detector (GCFID) using standard fatty acid mixture. Moisture values of hawthorn seeds varied between $14.49 \%-36.33 \%$. 10 fatty acid compositions belonging to 7 hawthorn taxa were determined, the highest were linoleic (64.23\%), oleic (39.36\%) and palmitic acid $(8.16 \%)$ respectively.
\end{abstract}

KEY WORDS: Crataegus, volatile component, benzaldehyde, linoleic acid, Western Anatolia, Turkey

\section{INTRODUCTION}

\section{UVOD}

Turkey has a very rich flora with approximately 11.000 registered plant species (Davis et al., 1988; Güner et al.; 2000,
Erik and Tarıkahya, 2004). Flora of Turkey is intriguing not only due to high number of species but also due to high number of endemic species (Ekim et al., 1989).

Various unnatural products have been and are entering our lives in addition to medicines (Rodopman, 1990). Strong

\footnotetext{
1 Sevgin Özderln, Muğla Sıtkı Koçman University, Truffle Application and Research Center, 48000, Muğla/Turkey

${ }^{2}$ Hüseyin Fakir, Süleyman Demirel University, Faculty of Forestry, Department of Forest Engineering, 32260 Isparta/Turkey

${ }^{3}$ İ. Emrah Dönmez, Süleyman Demirel University, Faculty of Forestry, Department of Forest Industrial Engineering, 32260, Isparta/Turkey

*Corresponding author: sevginozderin@mu.edu.tr
} 
reactions caused by synthetic medicines and pollution caused by pharmaceutical industry have made herbal treatment important today (Bulut, 2006). Plants used as herbal drugs in treatment in traditional and modern medicine are called „Medicinal Plants” (Baydar, 2007). Turkey has a high number of medicinal and aromatic plants due to its rich flora. Plants have been used by local people for treatment purposes, as food, tea, spices, dye, insecticide, for treatment of animal diseases, as resin, glue and as volatile fixed oils, beverage and for cosmetics industry as a part of our long traditional cultural diversity (Faydaoğlu and Sürücüoğlu, 2011).

Pharmacologic studies carried out on these medicines based on their purpose of use have scientifically explained some biological effects (Baydar, 2007). Plants are characteristically composed of mostly high-molecular-weight substances such as carbohydrates, oil, protein, cellulose, lignin and pectin, which are called primary metabolites. Apart from primary metabolites which have functions that are essential to growth and development plants also contain small moleculare weight secondary metabolites such as alkaloids, volatile oils, glycosides, heterocyst, steroids, flavonoids, tannins, phenols, color substances and resins, which are sometimes at unme- asurable levels and are not essential in terms of the vitality of plants. Therapeutic activity of a medicinal plant arises from these bioactive substances. Plants which are rich in terms of secondary metabolites mostly fall into the group of medical and aromatic plants (Baydar, 2007).

Certain parts (leaf, flower, sprout, fruit, root) of hawthorn (Crataegus) species, which are one of the widely used plants among the public, are traditionally used to treat various diseases. Dried flower and fruits of hawthorn are prepared as tea and used to treat tonsillitis, coughing, poor coronary activity, cardiac pain, tachycardia, renal diseases, arteriosclerosis, liver pain and hemorrhoids (Baytop, 1984; Karadeniz, 2004; Meriçli, 1994). Hawthorn, which has been used as traditional medicine, has drew attention from the world of science and various studies have been carried out. Previous studies reported that different taxa of hawthorn have antiarrhythmic effect (Garjani et al., 2000); hypotensive effect and increase coronary blood flow (Birman et al., 2001), they have anti-ischemic (Al Makdessi et al., 1999) anti-inflammatory (Bor et al., 2012), antioxidant effect (Bahorun et al., 1994; Bahorun et al., 1996; Bor et al., 2012) they protect vascular integrity (Miller, 1998), have antiviral (Shahat

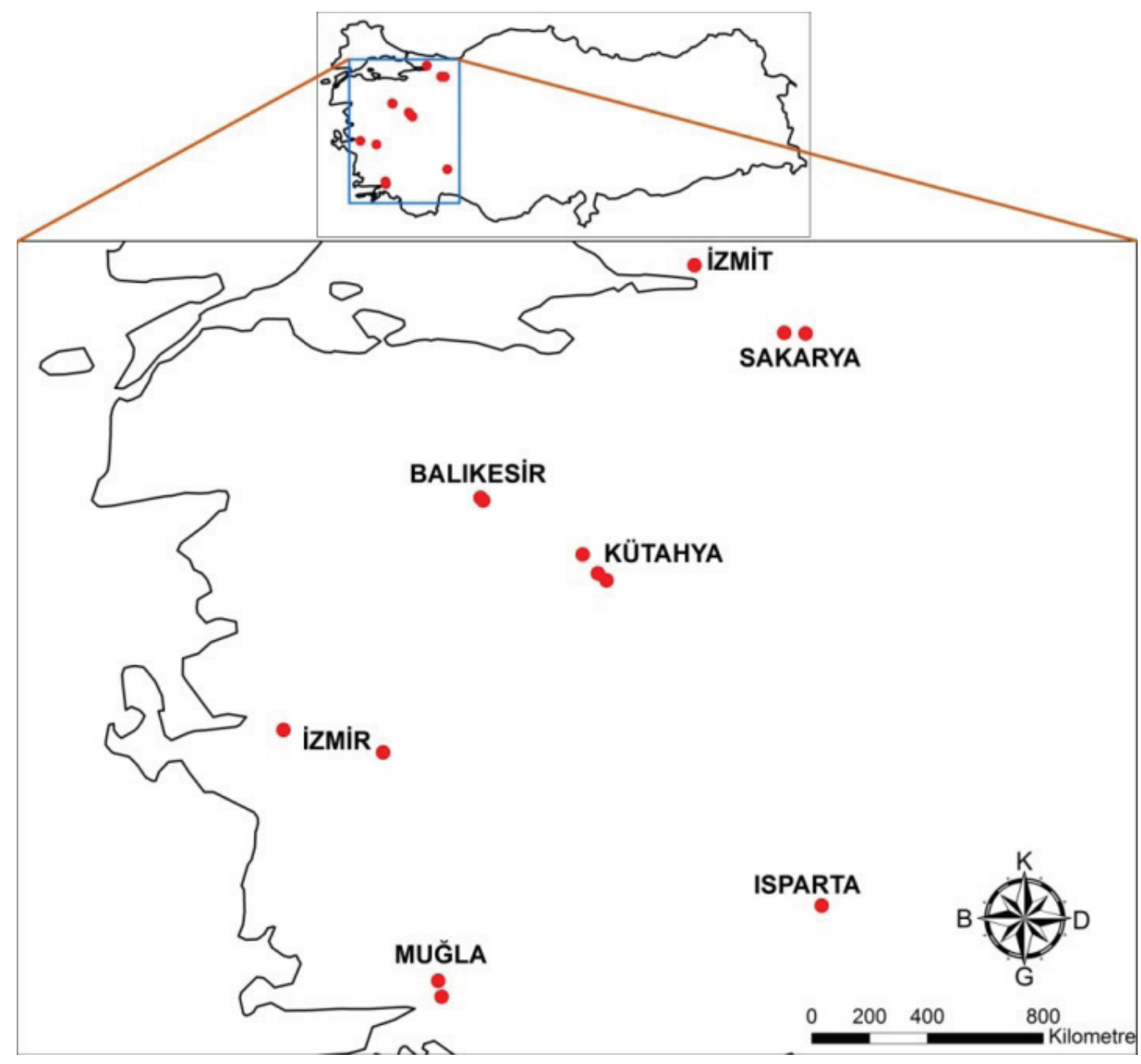

Figure 1. Study Area

Slika 1. Istraživano područje 
et al., 1998), antithrombotic (Arslan et al., 2011), antifungal (Orhan et al., 2007), antinociceptive (Bor et al., 2012) effects and that they are effective in early periods of congestive heart failure (Rietbrock, 2001).

Hawthorn refers to deciduous trees or shrubs belong to genus Crataegus L. of family Rosaceae which generally has thorns (Christensen, 1992; Dönmez, 2004). Genus hawthorn has around 200 species around the world. Although more than 1000 names have been reported for this plant around the world, it has 100-200 species. Some characteristics of the genus such as leaf and seed morphology, number of seed and fruit color are polymorphous. Polymorphism and hybridization are the reason for high number of synonyms. There are 26 hawthorn taxa growing in Turkey (Dönmez, 2007).

This study aims to determine chemical contents of different hawthorn species which are a wild species distributed in Western Anatolia.

\section{MATERIAL AND METHOD MATERIJAL I METODA}

Material of the study consisted of Crataegus samples collected from A1-A2, A3,B1-B2, B3, C2 and C3 squares according to squaring system in Flora of Turkey (Davis et. al., 1988) in 2010-2014 period (Figure 1, Table 1). Field study was conducted in the study area in blooming (May-June), fruit ripening (September-October) periods of Crataegus species. Samples were collected and registered and plant samples were maintained at Forest Botanic Laboratory of
Süleyman Demirel University, Faculty of Forestry for drying and storage according to herbarium techniques. Plants were identified and kept at Süleyman Demirel University, Faculty of Forestry herbarium.

\section{Determination of volatile components - Određivanje hlapljivih komponenti}

Volatile components of hawthorn leaves and flowers were determined according to solid phase microextarction method (Vichy et. al., 2003). Collected plant materials were dried at room temperature. $2 \mathrm{gr}$ of each sample were weighted and placed in glass bottles and were heated at $60 \mathrm{C}^{0}$ for $15 \mathrm{mi}$ nutes. Then they were soaked in injector bottle with a suitable fiber tip and absorbed for $30 \mathrm{~min}$. The compounds that were exposed to fiber tip were injected to injection block of GC unit and were kept for $5 \mathrm{~min}$ for absorption.

GC-MS device (Shimadzu QP 5050, Japan) was used to determine volatile components. HP-5 MS ( $30 \mathrm{~m} \mathrm{x} 0.25 \mathrm{~mm}$ long and 0.25 um film thickness) column and Helium (10 psi flow rate) was used as carrier gas in the device. Injection block temperature was $240^{\circ} \mathrm{C}$ and detector temperature was $250^{\circ} \mathrm{C}$.

\section{Determination of fruit seed fixed oil composition - Određivanje sastava nehlapljivih ulja u sjemenkama voća}

GC-MS (with FID Detector, Shimadzu QP 5050, and Japan) in Suleyman Demirel University Experimental and Observational Student Research and Application Center was used

Table 1. Locations of study material

Tablica 1. Lokacije od promatrani materijal

\begin{tabular}{|c|c|}
\hline $\begin{array}{l}\text { Taxa } \\
\text { Takson }\end{array}$ & $\begin{array}{l}\text { Locations and elevations } \\
\text { Lokacije i uzvisine }\end{array}$ \\
\hline $\begin{array}{l}\text { C. pentagyna Waldst. \& Kit. ex Willd. } \\
\text { subsp. Pentagyna }\end{array}$ & İzmit province locality around TaşKöprü warehouse 456 m. \\
\hline $\begin{array}{l}\text { C. orientalis Pall. ex M. Bieb. } \\
\text { subsp. Orientalis }\end{array}$ & $\begin{array}{c}\text { Sakarya province Taraklı county; Karagöl Uğurlu plateau locality } 1385 \text { m. } \\
\text { Balıkesir province Dursunbeyli county Aktuzla locality } 1510 \text { m. } \\
\text { İzmir province Kemal Paşa county Ayrancı locality } 820 \text { m. } \\
\text { İzmir province Ödemiş county Gölcük locality1120 m. } \\
\text { Kütahya province Emet county Tahtalı locality } 1120 \text { m. }\end{array}$ \\
\hline $\begin{array}{l}\text { C. orientalis Pall. ex M. Bieb. } \\
\text { subsp. szovitsii (Pojark.) K. I. Chr }\end{array}$ & Kütahya province Emet county Alıç locality 1450 m \\
\hline Crataegus tanacetifolia (Lam.) Pers. & $\begin{array}{l}\text { Kütahya province Emet county Çomar locality } 1450 \text { m. } \\
\text { Sakarya province Taraklı county, Esenyurt plateau } 1227 \text { m, }\end{array}$ \\
\hline Crataegus azarolus L. var. aronia & $\begin{array}{c}\text { Muğla province Yaraş village Akyaka locality } 812 \mathrm{~m} . \\
\text { Isparta Kovada Lake locality } 933 \mathrm{~m} .\end{array}$ \\
\hline $\begin{array}{l}\text { Crataegus monogyna Jacq. } \\
\text { var. lasiocarpa (Lange) K. I. Christ }\end{array}$ & $\begin{array}{l}\text { Balıkesir province Dursunbeyli county Aktuzla } \\
\text { locality } 1510 \mathrm{~m} .\end{array}$ \\
\hline $\begin{array}{l}\text { Crataegus monogyna Jacq. } \\
\text { var. monogyna }\end{array}$ & Muğla province Ula county Çiçekli locality 250 m. \\
\hline
\end{tabular}


Table 2. Leaf and flower volatile oil components of hawthorn taxa (\%)

Tablica 2. Hlapljive komponente ulja iz lista i cvijeta taksona gloga (\%)

\begin{tabular}{|c|c|c|c|c|c|c|c|c|c|c|c|c|c|c|}
\hline \multirow[b]{2}{*}{$\begin{array}{l}\text { LRI } \\
\text { LRI }\end{array}$} & \multirow[b]{2}{*}{$\begin{array}{l}\text { Components } \\
\text { Komponente }\end{array}$} & \multicolumn{13}{|c|}{ Volatile oil component (\%) } \\
\hline & & 1 & 2 & 3 & 4 & 5 & 6 & 7 & 8 & 9 & 10 & 11 & 12 & 13 \\
\hline 650 & Isopropyl acetate & & & & 1,54 & 0,70 & & & 1,88 & & & & & \\
\hline 662 & Butyraldehyde & 5,54 & 17,27 & 9,83 & 9,03 & 0,55 & 15,7 & 30,93 & 8,81 & 16,21 & 15,21 & 4,49 & 36,40 & 38,27 \\
\hline 680 & Ethylvinyl carbinol & 1,62 & 7,13 & 4,35 & 0,52 & & 3,22 & 6,26 & & 2,87 & 4,01 & & 1,62 & 0,90 \\
\hline 682 & Propyl methyl ketone & & & & & 6,24 & & & & & & & & \\
\hline 696 & Valeraldehyde & & 4,23 & 1,54 & 0,64 & & 1,28 & 3,04 & 2,75 & 3,86 & 3,95 & 0,40 & 0,59 & 0,93 \\
\hline 697 & Diethyl ketone & & & & & 0,79 & & & & & & & & \\
\hline 703 & Furan & 2,48 & & & 1,06 & 0,50 & 3,6 & & & 0,14 & 0,21 & 0,29 & 2,96 & 1,94 \\
\hline 729 & Isoamyl alcohol & 4,74 & 5,41 & 7,45 & & 1,20 & 1,8 & 3,8 & & 2,51 & 3,45 & 1,43 & 1,83 & 3,52 \\
\hline 732 & Formate & 1,09 & 2,97 & 3,64 & & 0,80 & 0,96 & 3,92 & & 1,46 & 1,97 & & 0,57 & 1,62 \\
\hline 751 & 2-Pentenal & 0,61 & 0,63 & 0,91 & & & 0,87 & 1,16 & & 0,76 & 1,62 & 0,05 & 0,40 & 0,51 \\
\hline 761 & 1-pentanol & 0,59 & 0,71 & 0,54 & & & 0,74 & 0,72 & & 1,35 & 0,45 & 0,06 & 0,41 & \\
\hline 767 & 2-Pentenol & 0,76 & 1,17 & 0,91 & & & 1,16 & 0,58 & & 1,33 & 0,75 & 0,10 & 0,78 & 0,84 \\
\hline 801 & Capronaldehyde & 16,55 & 5,10 & 6,05 & 2,60 & 2,60 & 18,13 & 12,55 & & 12,26 & 4,28 & 1,94 & 10,16 & 11,14 \\
\hline 845 & Furfural & & & & & & & & & & & & & 0,06 \\
\hline 850 & 2-Hexenal & 18,70 & 7,05 & 12,96 & 12,68 & 12,15 & 13,11 & 13,28 & 4,95 & 13,73 & 21,67 & 2,57 & 17,01 & 8,29 \\
\hline 853 & 3-Hexenol & 12,47 & 4,94 & 5,53 & 1,84 & 2,03 & 4,53 & 4,85 & & 7,00 & 9,12 & 0,40 & 6,37 & 2,82 \\
\hline 866 & 2-Hexenol & & & & 0,69 & 1,03 & & & & & & & & \\
\hline 867 & Hexanol & 21,10 & 11,88 & 4,18 & 2,99 & 11,85 & 9,74 & 4,76 & 2,57 & 20,10 & 1,92 & 2,52 & 4,09 & 0,95 \\
\hline 878 & 2,6-Lutidine & & & & 1,05 & 0,69 & & & 0,83 & & & & & \\
\hline 891 & Styrene & & & & 3,05 & 3,31 & & & 2,79 & & & & & \\
\hline 898 & 2-Heptanone & & 0,34 & 0,15 & & & & & & & & & & \\
\hline 902 & 4-Heptenal & 0,10 & & & & & & & & & 0,04 & & 0,06 & 0,06 \\
\hline 906 & Enanthaldehyde & 0,15 & & & 3,52 & 0,48 & 0,06 & & 1,89 & 0,13 & & & 0,15 & 0,30 \\
\hline 914 & Sorbic aldehyde & 0,16 & & & & & 0,18 & & & & & & & \\
\hline 933 & $\alpha$-Pinene & 0,19 & 0,88 & 18,68 & 2,27 & 1,55 & 0,56 & 0,72 & 3,34 & 0,32 & 0,60 & 0,04 & 0,35 & 0,17 \\
\hline 934 & 3-Heptanone & & & & & & & & & & & 1,23 & & \\
\hline 957 & Camphene & & & 0,09 & & & & & & & & & & \\
\hline 964 & Benzaldehyde & 8,34 & 17,68 & 11,46 & 4,97 & 8,06 & 6,30 & 6,31 & 4,55 & 10,83 & 23,86 & 82,54 & 10,31 & 17,09 \\
\hline 970 & n-Heptanol & & & & 1,87 & 1,30 & & & 2,22 & & & & & \\
\hline 978 & $\beta$-Pinene & & & 1,53 & & & & & & & 0,07 & & & \\
\hline 982 & Vinyl amyl carbinol & & 0,26 & 0,12 & & & & 1,45 & & 0,10 & & & & 0,08 \\
\hline 986 & Hept-5-en-2-one & 0,51 & 0,60 & 0,50 & 1,56 & 2,98 & 1,71 & 0,72 & 1,04 & 0,71 & 0,58 & 0,17 & 0,43 & 0,58 \\
\hline 989 & Hexyl menthyl ketone & & 1,04 & & & & & & & & & & & \\
\hline 991 & Myrcene & & & 2,75 & 3,91 & 4.32 & & & 5.46 & & & & & \\
\hline 997 & Trans-2-(2 pentenyl)furan & 0,19 & & 0,20 & & & 0,13 & & & 0,12 & 0,20 & 0,22 & 0,27 & 0,66 \\
\hline 1006 & Caprylaldehyde & & & & & 1.87 & 0,10 & & 1.81 & & & & 0,16 & \\
\hline 1009 & 3-delta-carene & 0,14 & 0,15 & 0,16 & 1,30 & 0,67 & 0,18 & & 1,47 & 0,09 & 0,17 & 0,21 & 0,22 & 0,14 \\
\hline 1013 & Heptandienal & 1,19 & 0,22 & 0,19 & & 0,47 & 0,08 & & & 0,25 & 0,14 & 0,08 & 0,93 & 0,18 \\
\hline 1012 & Hexanoic acid, 2-propenyl ester & 0,22 & & & & & & & & & & & 0,20 & \\
\hline 1018 & $\alpha$-Terpinene & & & & 0,67 & & & & 0,98 & & & & & \\
\hline 1025 & Cymene & 0,24 & 0,20 & 0,34 & 6,48 & 4,95 & 0,28 & & 7,19 & & 0,21 & 0,14 & 0,26 & 0,30 \\
\hline 1030 & Limonene & 0,15 & 0,22 & 1,39 & 4,39 & 3,95 & 0,20 & & 5.31 & 0,12 & 0,19 & 0,21 & 0,36 & 0,64 \\
\hline 1032 & Eucalyptol & 0,08 & 0,31 & & 3,52 & 1,90 & 0,12 & 0,32 & 5,22 & 0,15 & 0,27 & 0,05 & 0,26 & 0,18 \\
\hline 1040 & Benzyl alcohol & 0,23 & 0,26 & & & 0,69 & & & & & 0,19 & & 0,24 & 0,16 \\
\hline 1045 & Phenylacetaldehyde & 0,30 & 0,13 & 0,33 & & 0,94 & 0,12 & 0,25 & 0,64 & 0,16 & 0,26 & & & 1,37 \\
\hline 1046 & $\beta$-ocimene & & & & & 1,47 & & & & & & & & \\
\hline 1050 & Nonylol & & & & & & & & & & & 0,11 & & \\
\hline 1058 & $\gamma$-Terpinene & & & & 2,25 & 1,19 & & & 3,58 & & & & & \\
\hline 1084 & Benzyl mercaptan & & & & 1,40 & & & & & & & & & \\
\hline 1059 & 2-Octenal & & & & & & & & & & & & & 0,12 \\
\hline 1068 & Acetophenone & & & & & & & & & & & & & 0,36 \\
\hline 1098 & Terpinolene & 0,07 & 0,14 & 0,09 & 1,13 & 0,65 & & 0,28 & 1,26 & & & 0,05 & 0,08 & 0,33 \\
\hline 1101 & Undecane & & & 0,05 & & & & & & & & & & 0,08 \\
\hline 1107 & Pelargonaldehyde & 0,20 & 0,18 & 0,09 & & 0,59 & 0,15 & & & 0,27 & 0,27 & 0,05 & 0,67 & 1,58 \\
\hline
\end{tabular}




\begin{tabular}{|c|c|c|c|c|c|c|c|c|c|c|c|c|c|c|}
\hline 1113 & Phenethyl alcohol & 0,32 & 0,21 & 0,19 & & & 0,12 & & & 0,25 & & 0,07 & 1,12 & 0,59 \\
\hline 1153 & 2,6-Nonadienal & & & & & & & & & & & 0,05 & & 0,09 \\
\hline 1170 & 2-Nonenol & & & & & & & & & & & 0,27 & & 0,21 \\
\hline 1192 & Methyl salicylate & 0,21 & 1,84 & & & & 0,40 & & & & & 0,07 & 0,13 & 0,15 \\
\hline 1198 & $\alpha$-Terpineol & & 0,50 & & & & & & 0,59 & & & & & 0,10 \\
\hline 1243 & Hexyl3methylbutanoate & & & & & & 0,22 & & & 0,25 & & & & 0,10 \\
\hline 1257 & p-Anisaldehyde & & 0,57 & & & & & & & 0,41 & & 0,08 & 0,40 & 1,84 \\
\hline 1294 & $\begin{array}{l}\text { menthyl Nonyl ketone, } \\
\text { undecan }\end{array}$ & & 3,66 & & & & & & 3,05 & 0,22 & & 0,09 & & 0,21 \\
\hline 1300 & Tridecane & & & & 0,53 & 1,16 & & & & & & & & \\
\hline 1307 & octyl propanoate. & & & & 0,57 & & & & & & & & & \\
\hline 1357 & Eugenol & & & & & & & & 0,61 & & & & & \\
\hline 1375 & $\alpha$-Copaene & & 0,21 & & & & 1,29 & & & 0,39 & 0,24 & & & \\
\hline 1382 & $\beta$-Bourbonene & 0,09 & 0,64 & & & & 2,85 & & 0,68 & & 0,42 & & & \\
\hline 1400 & Tetradecane & & & & 1,37 & 2,96 & & & 1,44 & & & & & 0,12 \\
\hline 1418 & $\beta$-Caryophyllene & 0,44 & 0,79 & 0,61 & 1,38 & & 8,11 & 3,29 & 1,48 & 0,87 & 3,29 & 0,02 & 0,15 & 0,06 \\
\hline 1423 & $\beta$-Cedrene & & 0,16 & & & & 0,99 & & & & & & & \\
\hline 1432 & trans- $\alpha$-Bergamotene & & & & & & & 0,20 & & & & & & \\
\hline 1454 & $\alpha$-Humulene & 0,07 & & & & & 0,37 & 0,15 & & & 0,17 & & & \\
\hline 1480 & 1,6,Cadinadiene & & & & & & 0,16 & & & & & & & \\
\hline 1495 & $\begin{array}{l}\text { Menthyl undecyl ketone, } \\
\text { tridecan }\end{array}$ & & & & & & & & 1,26 & & & & & \\
\hline 1504 & $\alpha$-farnesene & & 0,18 & & 5,68 & 5,90 & 0,44 & 0,46 & 4,58 & & 0,22 & & & \\
\hline 1581 & Hexyl benzoate & 0,06 & & & & & 0,14 & & & 0,44 & & & & \\
\hline 1582 & Hexadecane & & & & 1,44 & 1,12 & & & 0,64 & & & & & \\
\hline 1900 & Nonadecane & & & & 2,64 & 0,52 & & & 1,08 & & & & & \\
\hline 1215 & $\beta$-cyclocidral & 0,10 & & & & & & & & & & & & \\
\hline 2100 & Heneicosane & & & & 0,67 & & & & & & & & & \\
\hline
\end{tabular}

***LRI: Retention index

Leaf and flower volatile components Sample 1. C. pentagyna subsp. pentagyna, sample 2. C. orientalis subsp. orientalis, sample 3. C. orientalis subsp. orientalis, sample 4.C. orientalis subsp. orientalis, sample 5. C. orientalis subsp. orientalis, sample 6. C. orientalis subsp. orientalis, sample 7. C. orientalis subsp. szovitsii, sample 8. C. tanacetifolia, sample 9. C. tanacetifolia, sample 10. C. azarolus var. aronia, sample 11. C. azarolus var. aronia, sample 12. C. monogyna var. lasiocarpa, sample 13. C. monogyna var. monogynyna.

Table 3. FID results of seed fatty acid components of hawthorn taxa

Tablica 3. Rezultati plameno-ionizacijskog detektora za komponente masnih kiselina u sjemenkama taksona gloga

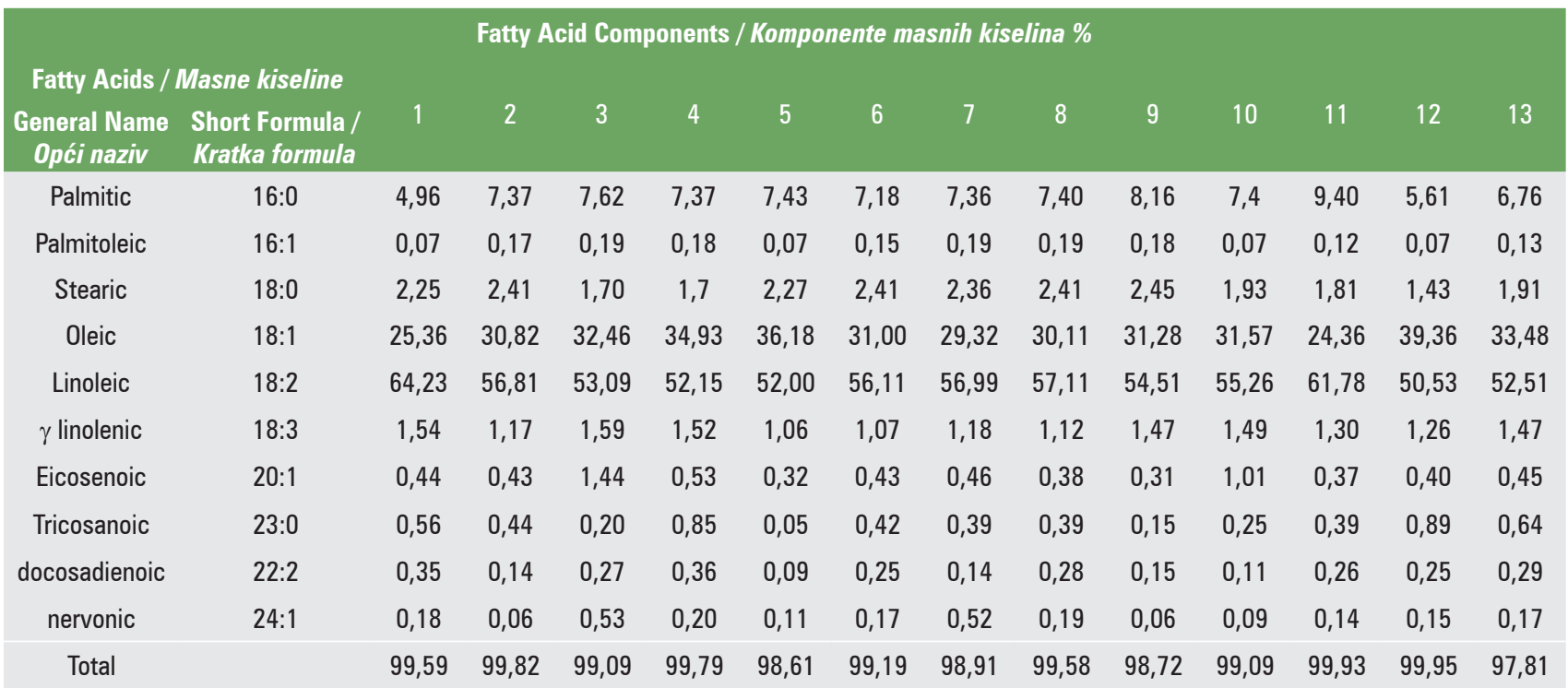

Seed fatty acids results Sample 1. C. pentagyna subsp. pentagyna, sample 2. C. orientalis subsp. orientalis, sample 3. C. orientalis subsp. orientalis, sample 4. C. orientalis subsp. orientalis, sample 5. C. orientalis subsp. orientalis, sample 6. C. orientalis subsp. orientalis, sample 7. C. orientalis subsp. szovitsii, sample 8.C. tanacetifolia, sample 9.C. tanacetifolia, sample 10. C. azarolus var. aronia, sample 11. C. azarolus var. aronia, sample 12. C. monogyna var. lasiocarpa, sample 13.C. monogyna var. monogyna species 
to determine seed fatty acid amount and composition by injecting the samples to the device. Hexane was used to obtain fixed oil by cold extraction (Nimal Ratnayake et. al., 2006). After 24 hours, pure oil was separated from hexane using rotary evaporator and fixed oil was extracted. Pure cellulose from the extracted fixed oil was exposed to cold extraction in hexane by mixing approximately $15 \mathrm{~g}$ sample placed in cartridge at certain intervals for 24 hours. Then, hexane was removed from rotary evaporator, thus leaving oil extracts. $100 \mu \mathrm{L}$ of the extract was kept in derivatizing agent containing $0.5 \%$ sodium methoxide (80:20 (methanol: isooctane) at room temperature for 24 hours $(25 \mathrm{c}) .1$ $\mathrm{mL}$ isooctane was added and mixed at vortex device (AOCS, 2005). The supernatant was let to separate. $1 \mu \mathrm{L}$ of supernatant was injected to GC- FID. Chromatogram evaluation was determined according to supelco 37-fame mixture (fatty acid mixture) standard retention time.

\section{RESULTS}

REZULTATI

Volatile components and contents of C. pentagyna Waldst. \& Kit. ex Willd. subsp. pentagyna, C. orientalis Pall. ex M. Bieb. subsp. orientalis, C. orientalis Pall. ex M.Bieb. subsp. szovitsii (Pojark.) K.I.Chr, Crataegus tanacetifolia (Lam.) Pers., Crataegus azarolus L. var. aronia, Crataegus monogyna Jacq. var. lasiocarpa (Lange) K.I.Christ, Crataegus monogyna Jacq. var. monogyna taxa

that are naturally distributed in Western Anatolia were determined by SPME (Solid-Phase Microextraction method). Results of volatile components are presented in Table 2

Seed fatty acid components of hawthorn taxa were determined in GC-FID analyses. The results for these samples are presented in Table 3.

\section{DISCUSSION AND CONCLUSION RASPRAVA I ZAKLJUČAK}

A total of 81 components were determined in volatile oils of leaves and flowers of 7 Crataegus taxa collected from different localities. Major components are Benzaldehyde, butyraldehyde, 2 -hexenal $(82.54 \%, 38.27 \%$ and $21.67 \%$ respectively). The high content of Benzaldehyde, butyraldehyde, 2-hexenal and hexanol were determined in volatile oils of C. orientalis subsp. orientalis samples collected from different localities.

Kovaleva et al., (2009) analyzed chemical composition of volatile oils extracted from the flowers of C. jackii, C. robesonianave C. flabellata. Researchers determined main components of $C$. robesoniana as phthalate (15.62\%), squalene (13.08\%), tricosane (11.11\%), main components of $C$. flabellata as tricosane (19.21\%), heneicosane (12.59\%), nona- cosane (11.22\%) and main volatile components of C. jackii as tricosane $(17.88 \%)$, and phthalate $(13.38 \%)$ heneicosene-1 (12.53\%). This indicates that different components can be determined in different taxa.

Fruit seed samples of 7 Crataegus taxa were analyzed and 10 fatty acid components belonging to each taxon were determined. Linoleic (18.2\%), oleic (18.1\%) and palmitic (16.0\%) acid components were the main acids present. Linoleic acid, which was found at the highest ratio (64.23\%) was determined from C. pentagyna subsp. pentagyna sample collected from Taşköprü locality.

Barros et al., (2010) analyzed fatty acid composition of flower and fruits of C. monogyna Jacq. and determined the highest content of linoleic acid in unripe fruits $(58.5 \%)$; ripe fruits $(17.53 \%)$, flower buds (15.64\%), flowers (14.17\%) over ripened fruits (13.12\%). The researchers reported that the fatty acid component with the second highest content was tricosanoic acid, which was determined in flower buds (36.95\%), flowers (33.67\%), unripe fruits $(8.18 \%)$, ripened fruits $(32.77 \%)$ and over unripened fruits (30.40\%). $\gamma$ linoiec acid was reported to be the $3 \mathrm{rd}$ fatty acid with the highest content, which was determined in flower buds (26.79\%), flowers (29.51\%), unripe fruits (5.98\%), ripened fruits $(7.41 \%)$, and over ripened fruits (15.65\%). The high content of palmitic acid respectively in over ripened fruits (15.52\%), ripened fruits (13.73\%), flowers (11.23\%), flower buds (11.02\%), and unripe fruits (10.61\%).

Hawthorn fruits have a wide field of use in its distribution areas and thus around the world. Hawthorn, which has a significant role in food industry, is directly consumed as fruit. Furthermore, due to chemicals such as fatty acid, sterols etc. in its fruit, a review of the literature has shown that hawthorn has antioxidant, antiviral, antifungal and anti-inflammatory effects (Bahorun et al.,1994; Shahat et al., 1998; Orhan et al., 2007; Ahumada et al., 1997). This study directly concentrated on seeds of hawthorn, which is consumed as food, after removal of flesh section. Thus, fatty acid compositions of fruit seeds, which are considered as waste after consumption of fruits, were identified and amounts of fatty acids were determined in terms of chemical use. The fatty acid with the highest procentage in all samples was found to be linoleic acid. Furthermore, other fatty acids were determined at significant amounts.

\section{ACKNOWLEDGEMENTS}

\section{ZAHVALE}

We express our sincere appreciation to Suleyman Demirel University, Coordinatorship of Scientific Research Projects for their financial support by project which numbered as 3211-D2-12. 


\section{REFERENCES}

\section{LITERATURA}

- Al Makdessi, S., Sweidan, H., Dietz, K., Jacob, R. 1999. Protective effect of Crataegus oxyacantha against reperfusion arrhythmias after global no-flow ischemia in the rat heart, Basic research in cardiology, 94,(2), 71-77p.

- AOCS Method Ce 1h-05, „Determination of cis-, trans-, Saturated, Monounsaturated and Polyunsaturated Fatty Acids in Vegetable or Non-ruminant Animal Oils and Fats by Capillary GLC” AOCS Official Methods 2005. American Oil Chemists Society.

- Arslan R, Bor Z, Bektas N, Mericli A.H., Ozturk Y. 2011. Antithrombotic effects of ethanol extract of Crataegus orientalis in the carrageenan-induced mice tail thrombosis model. Thromb Res 127: 210-213.

- Ahumada, C., Saenz, T., Garcia, D., De La Puerta, R., Fernandez, A., Martinez, E. 1997. The effects of a triterpene fraction isolated from Crataegus monogyna Jacq. on different acute inflammation models in rats and mice. Leucocyte migration and phospholipase A2 inhibition, Journal of pharmacy and pharmacology, 49,(3), 329-331.

- Barros, L., Carvalho, A.M., Ferreira, I.C.F.R. 2010. Comparing the composition and bioactivity of Crataegus monogyna flowers and fruits used in folk medicine. Phytochemical Analysis, 22, 181-188.

- Baydar, H., 2007. Science and Technology of Medicinal, Aromatic and Joy Plants, Süleyman Demirel University Faculty of Agriculture, S.D.U. Publication No: 51, 216 p.

- Baytop, T. 1984. Treatment with plants in Turkey. Istanbul University Publication No. 3255, Istanbul Türkiye).

- Bahorun, T., Trotin, F., Pommery, J., Vasseur, J., Pinkas, M. 1994. Antioxidant activities of Crataegus monogyna extracts, Planta medica, 60,(4), 323-323.

- Bahorun, T., Gressier, B., Trotin, F., Brunet, C., Dine, T., Luyckx, M.. 1996. Oxygen species scavenging activity of phenolic extracts from hawthorn fresh plant organs and pharmaceutical preparations, Arzneimittel-Forschung, 46,(11), 1086-1089.

- Birman, H., Tamer, S., Melikoglu, G. 2001. Hypotensive activity of Crataegus tanacetifolia, Journal of Pharmacy of Istanbul University, 34,(2), 23-26.

- Bor, Z., Arslan, R., Bektas, N., Pirildar, S., Dönmez, A. A. 2012. Antinociceptive, antiinflammatory, and antioxidant activities of the ethanol extract of Crataegus orientalis leaves. Turk J Med Sci, 42 (2), pp.315-324.

- Christensen, K.I. 1992. Revision of Crataegus sect. Crataegus and Nothosect. Crataeguineae (Rosaceae-Maloideae) in the Old World.Syst. Bot. Monograms, 35: 1-199.

- Davis, P.H., Mill, R.R., Tan, K. 1988. Flora of Turkey And East Aegean Islands, (Supplements I), Vol. 10, Edinburgh University Press, Edinburgh.

- Dönmez, A.A. 2004. The Genus Crataegus L. (Rosaceae) with Special Reference to Hybridisation and Biodiversity in Turkey, Turk. J. Bot.,28, 29-37.
- Dönmez, A.A. 2007. Taxonomic note on the genus Crataegus(Rosaceae) in Turkey. Bot. J. Linnean Soc., 155: 231240.

- Ekim, T., Koyuncu, M., Erik, S., İlarslan, R. 1989. Rare and Endemic Plants of Turkey, Turkish Association for Conservation of Nature and Natural Resources, Publication no: 18, Ankara.

- Erik, S., Tarıkahya, B. 2004. About Flora of Turkey, Kebikeç, 17, 139-163.

- Faydaoğlu, E., Sürücüoğlu, M,S. 2011. History of the Use of Medical and Aromatic Plants and their Economic Importance. Kastamonu University, Journal of Forestry Faculty, 11(1):52-67.

- Garjani, A., Nazemiyeh, H., Maleki, N.,Valizadeh, H. 2000. Effects of extracts from flowering tops of Crataegus meyeri A. Pojark. on ischemic arrhythmias in anaesthetized rats, Phytotherapy Research, 14,(6), 428-431.

- Güner, A., Özhatay, N., Ekim, T., Başer, K.H.C. (eds) 2000. Flora of Turkey and the East Aegean Islands. Suppl. II, Edinburgh University. Press, Edinburgh

- Karadeniz, T. 2004. Curative Fruits. Karadeniz Technical University Ordu Faculty of Agriculture Department of Horticulture, Ordu, 34-36.

- Kovaleva A.M, Goncharov N.F., Komissarenko, A.N. 2009. GC/ MS study of essential oil components from flowers of C. jackii, C. robesoniana and C. flabellate. Chem Nat Compd 45: 582-584.

- Meriçli, A.H. and Ergezen K. 1994. Flavonoids of Crataegus tanacetifolia (Lam.) Pers. (Rosaceae) an endemic species from Turkey, Sci Pharm. 62, 277-281.

- Miller, A. 1998. Botanical influences on cardiovascular disease, Alternative medicine review: a journal of clinical therapeutic, 3,(6), 422-431

- Nimal Ratnayake, W. M., Hansen, S. L., Kennedy, M. P. 2006. Evaluation of the CP-Sil 88 and SP-2560 GC Columns Used in the Recently Approved AOCS Official Method Ce 1h-05: Determination of cis-, trans-, Saturated, Monounsaturated, and Polyunsaturated Fatty Acids in Vegetable or Non-ruminant Animal Oils and Fats by Capillary GLC Method JAOCS, 83(6):475488.

- Rietbrock, N., Hamel, M., Hempel, B., Mitrovic, V., Schmidt, T.,Wolf, G. 2001. Actions of standardized extracts of Crataegus berries on exercise tolerance and quality of life in patients with congestive heart failure, Arzneimittel-Forschung, 51,(10), 793 p.

- Orhan, I., Özcelik, B., Karta,1 M., Ozdeveci, B., Duman, H. 2007. HPLC Quantification of vitexine-2-O-rhamnoside and hyperoside in three Crataegus species and their antimicrobial and antiviral activities. Chromatographia 66: S153-S157.

- Shahat, A., Ismail, S., Hammouda, F. 1998. Anti-HIV activity of flavonoids and proanthocyanidins from Crataegus sinaica. Phytomedicine 2: 133-136.

- Vichy, S., Castellote, A. I., Pizzale, L., Conte, L. S., Buxaderas, S., Lopez-Tamames, E. 2003. Analysis of virgin olive oil volatile compounds by headspace solid-phase microextraction coupled to gas chromatography with mass spectrometric and flame ionization dedection, Journal of Chromatography A 983:19-33. 


\section{Sažetak}

Cilj ovoga rada je određivanje kemijskih svojstava svojti Crataegus pentagyna podvrste pentagyna, C. orientalis podvrste orientalis, C. orientalis podvrste szovitsii, C. tanacetifolia, C. azarolus var. aronia, C. monogyna var. lasiocarpa, C. monogyna var. monogyna prirodno rasprostranjenih u Zapadnoj Anatoliji. Uzorci lista i cvijeta prikupljenih 2010. - 2014. u provincijama Zapadne Anatolije Izmit, Sakarya, Balıkesir, Izmir, Kütahya, Muğla i Isparta, kako bi se odredile hlapljive komponente, osušeni su na sobnoj temperaturi. Hlapljive komponente, dobivene metodom mikroekstrakcije u krutoj fazi (SPME) u središnjem laboratoriju Sveučilišta Süleyman Demirel, određeni su uređajem puni naziv. Ukupno je utvrđena 81 hlapljiva komponenta iz 7 taksona gloga. Od hlapljivih komponenta ulja, koje su otkrivene u najvećim omjerima, pronađene su komponente benzaldehida (82,54\%), butiraldehida $(38,27 \%)$ i (E)2-heksenala $(21,67 \%)$.

Određena je i vlažnost sjemenki uzoraka gloga, sakupljenih u uzorkovanim područjima tijekom perioda sazrijevanja. Određen je i sastav masnih kiselina pomoću uređaja GC-FID, koristeći se standardnom mješavinom masnih kiselina. Vlažnost sjemenki gloga varirala je između 14,49\% - 36,33\%. Identificirano je 10 sastava masnih kiselina iz 7 taksona gloga, od čega je najviše linoleinske kiseline (64,23\%), oleinske kiseline (39,36\%) i palmitinske kiseline $(8,16 \%)$.

KLJUČNE RIJEČI: Crataegus, hlapljive komponente, benzaldehid, linoleinska kiselina, Zapadna Anatolija, Turska 